\title{
VIBRATIONAL CHARACTERISTICS OF HARP SOUNDBOARDS
}

\author{
Chris Waltham, Andrzej Kotlicki, Laura Dunwoody, Tina Lee, Ju lia Lin and Billy Lin \\ Department of Physics \& Astronomy, University of British Columbia, Vancouver BC V6T 1Z1
}

\section{INTRODUCTION}

A concert harp (Fig. 1) is basically a triangular structure, formed of the post or (fore)pillar, the neck, and a soundboard mounted on a soundbox. The strings are attached at one end to tuning pegs and bridge-pins mounted in the neck, and at the other end to the soundboard. The structure has to be made strong enough to withstand a total string tension of about $12-20 \mathrm{kN}$. The soundboard (Fig. 2) is approximately trapezoidal in shape, around $1.4 \mathrm{~m}$ in length, $0.5 \mathrm{~m}$ wide at the base, $0.1 \mathrm{~m}$ at the top, and of thickness varying from $11-12 \mathrm{~mm}$ at the bottom (bass) end to $2-2.5 \mathrm{~mm}$ at the top (treble). It is made of strips of spruce (Sitka or Engelmann) between 3 to $8 \mathrm{~cm}$ wide bonded together, and covered with a thin veneer, typically also of spruce. [1].

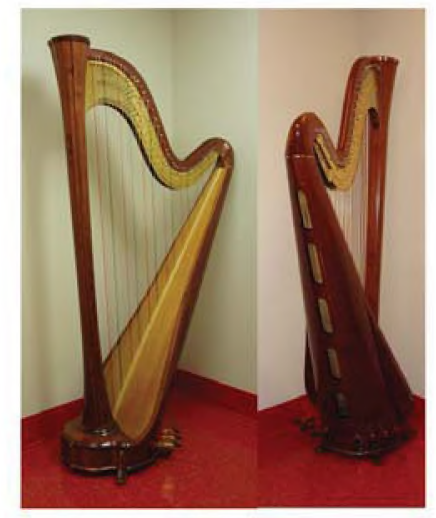

Fig. 1. The configuration of a modern 47-string concert harp, a Salvi Aurora (author photo).

The soundbox is semi-conical in shape, and is built up by bonding hardwood veneer (e.g. beech) around a mold. There are four or five soundholes in the back of the soundbox and one in the base. The primary function of these holes is to gain access to mount or replace strings, although they have important acoustical effects. Inside the back of the soundbox are strong U-shaped ribs (beech, aluminum, or steel) which prevent the box from undergoing too much flexure under the string tension.

A modern concert harp has 46 or 47 strings, running from $\mathrm{C} 1$ or D1 to G7. The lowest strings are mounted a few $\mathrm{cm}$ from the base of the soundboard, the highest strings a few $\mathrm{cm}$ from the top. The lower strings of concert harps are made of copper-wrapped steel, those in the mid-range are gut, and the upper strings are nylon.

\section{SOUND PRODUCTION}

Harp soundboxes, like those of all string instruments, are wooden shells with hole(s). This configuration, when properly optimized, ensures a wide frequency band with good radiativity and no frequencies where the radiativity is zero. The band stretches between two vibration modes. known as the "air" resonance and the "wood" resonance, often called A0 and T1 respectively. This phenomenon is well described in a classic work by Gabriel Weinreich [2]. A similar mechanism has been shown to operate in the harp at low frequencies [3].
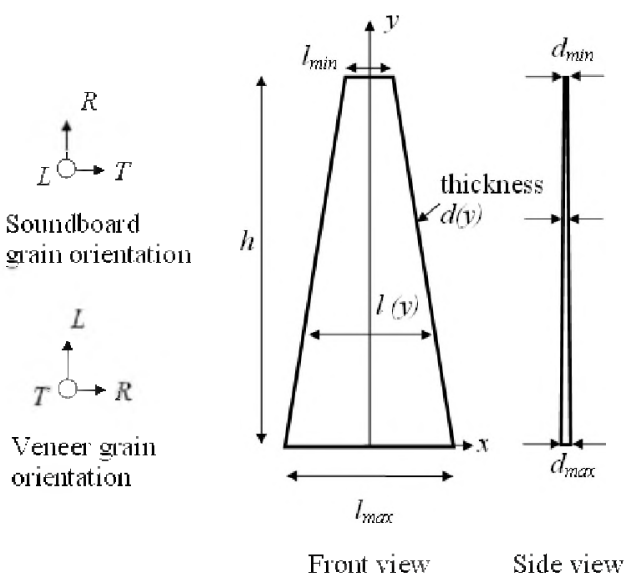

Fig. 2. Layout of harp soundboard, showing the $x-y$ coordinate system used in this paper, and the orientation of the wood grain for the soundboard base and veneer. The longitudinal $(L)$, transverse $(T)$ and radial $(R)$ directions refer to the natural cylindrical coordinate system of a tree limb.

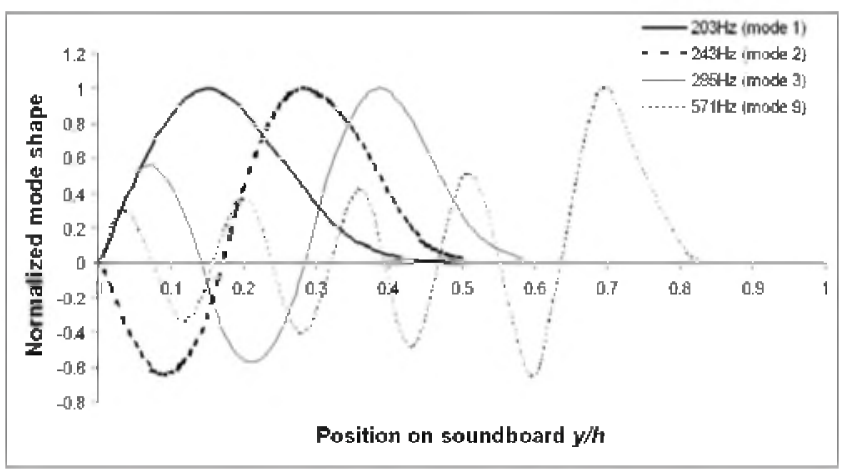

Fig. 3 Finite-element model predictions for the modal shapes for the first three modes of a trapezoidal spruce soundboard, clamped at all edges. The modal shapes are normalized such that the primary antinodes have unit amplitude.

The analysis in terms of $\mathrm{A} 0$ and $\mathrm{Tl}$ resonances only considers the lowest frequency behaviour of the soundboard. This is appropriate to the violin, whose strings excite the soundboard at the same point (via the bridge) where these modes are most prominent. In the case of the 
harp, the strings are attached all the way up the soundboard. and beyond the half-way mark, modes higher than the fundamental become dominant for sound production. The progression of modes of a bare soundboard, as calculated with finite-element techniques, is shown in Fig. 3.

\section{MEASUREMENTS AND ANALYSIS}

First, the soundboard was scanned by measuring the driving-point admittance at many points along the axis of symmetry, i.e. where the strings are attached. We make the assumption that the strings will primarily excite the symmetrical modes, ignoring for now second-order effects where non-planar string motion excites twisting modes. The admittance was measured using a small, light $(0.2 \mathrm{~g})$ accelerometer and an impact hammer.

The results for the admittance of the soundboard are shown in a contour plot (Fig. 4) in which the frequency is plotted against vertical position on the soundboard, and the shading represents the admittance (darker means higher). The progression in modal shapes is very plain, and the regions of highest admittance move steadily up the soundboard as the frequency increases. Some splitting is observed, particularly in mode $4(\sim 500 \mathrm{~Hz})$. Plotting the string pitch versus the attachment positions produces a "string trajectory" on the plot (marked by the points) which runs on the right side of the primary antinodes (except for the fundamental antinode - it runs past the left of that). Ref.[1] shows how important is the relationship between soundboard modes and the string frequencies at the string attachment positions.

Second, the admittance measurements were repeated with the accelerometer replaced by a microphone at the lowest sound hole on the back of the instrument. These results are plotted in Fig. 5 in the same manner as the admittances.

A comparison of Figs. 4 and 5 is striking. Where the progression of modes appear predominantly at single frequencies in the admittance plot, the sound pressure plot shows double resonances. This is particularly noticeable in the structures around 500 and $700 \mathrm{~Hz}$. A comparison with the low frequency work of Le Carrou [3] on a Camac Atlantide harp suggests that the two peaks near $200 \mathrm{~Hz}$ at the $400 \mathrm{~mm}$ position can be identified with the T1 and A0 resonances. At present this association is somewhat speculative as the Atlantide and Aurora harps do not have precisely the same specifications. However, if true, it is also likely that the other pairs of resonances have a similar phase relationship.

With a single microphone it was not possible unambiguously to show the phase relationships between all the pairs of peaks. These measurements need to be repeated with a velocity probe.

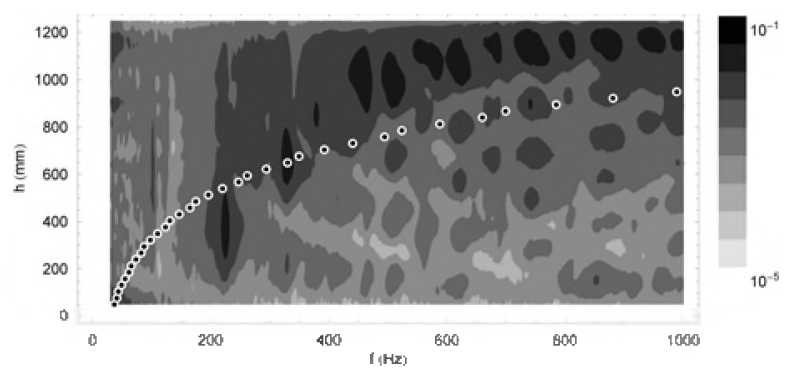

Fig. 4. Driving-point admittance data for the central string bar of a Salvi Aurora soundboard. The frequency is plotted against vertical position $h$ on the soundboard, and the shading represents the admittance $Y(\mathrm{~s} / \mathrm{kg})$. The points show the position and fundamental frequencies of the harp strings.

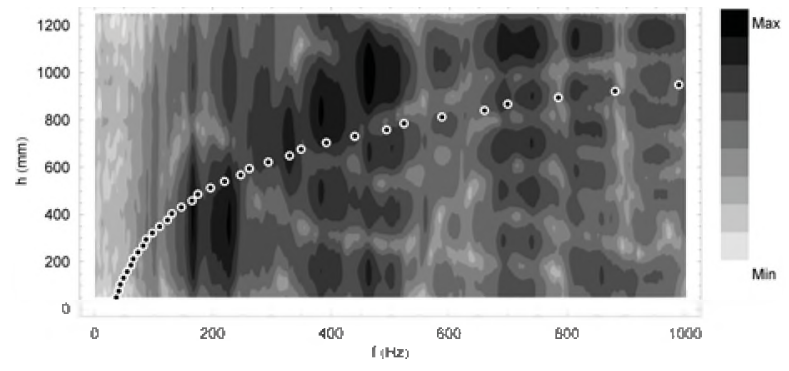

Fig. 5. Sound pressure at the lowest sound hole at the back of a Salvi Aurora, normalized to the force of an impact hammer striking position $h$ along the central string bar on the soundboard.

\section{CONCLUSION}

We are within striking distance of a fairly complete understanding of the vibro-acoustic behaviour of a modern harp soundbox. In the near future we hope to extend the work to earlier forms of the harp which had very different structures and distinctive voices.

\section{REFERENCES}

[1] Chris Waltham and Andrzej Kotlicki (2008). Vibrational characteristics of harp soundboards. JASA., 124, 1774-1780.

[2] G. Weinreich (1993). What science knows about violins, and what it doesn't know, Am. J. Phys. 61, 1067-1077

[3] J-L. Le Carrou, F. Gautier and E. Foltête (2007), Experimental studies of the $\mathrm{A} 0$ and $\mathrm{T} 1$ modes of the concert harp, $J A S A, 121,559-567$

\section{ACKNOWLEDGMENTS}

We thank Domenic DiTomaso and David Axen for technical assistance, and the University of British Columbia School of Music and Elizabeth Volpé-Bligh for making the Salvi Aurora concert harp available. 